Chirurg 2015 $\cdot 86: 409$

DOI 10.1007/s00104-014-2860-5

Online publiziert: 30. April 2015

๑) Springer-Verlag Berlin Heidelberg 2015

H.S. Hofmann ${ }^{1,2}$

${ }^{1}$ Abteilung für Thoraxchirurgie, Universitätsklinikum Regensburg

${ }^{2}$ Klinik für Thoraxchirurgie, Krankenhaus Barmherzige Brüder, Regensburg

\title{
Komplikationen in der Thoraxchirurgie
}

\section{Erfahrene Thoraxchirurgen berichten}

Der Begriff „Risikomanagement“ ist heute fast in allen Bereichen unseres Lebens gegenwärtig. Wenn Risiko als die Wahrscheinlichkeit für den Eintritt eines Schadens definiert wird, so ist im klinischen Risikomanagement die chirurgische Komplikation auch ganz eng und häufig mit einem Schaden für den Patienten verbunden. Daher ist vor allem der Risikominimierung sprich der Vermeidung von Komplikationen durch exzellente Prophylaxe höchste Aufmerksamkeit zu schenken. Eine Voraussetzung dafür ist, dass mögliche Komplikationen überhaupt bekannt sind.

Die Deutsche Gesellschaft für Thoraxchirurgie (DGT) hat im letzten Jahr während des 131. Kongresses der Deutschen Gesellschaft für Chirurgie (DGCH) vom 25. bis 28. März 2015 in Berlin ein Modellprojekt zur Risikominimierung bei Lungenoperationen inauguriert. In Zusammenarbeit mit dem Versicherer Ecclesia will die DGT Schadensfällen aus hunderten von Krankenhäusern der vergangenen 15 Jahren auswerten. Es soll analysiert werden, ob bestimmte Operationen oder Operationstechniken mit einem hohen Fehlerrisiko assoziiert sind. Am Ende des Projekts steht die Etablierung passgenauer Prophylaxeinstrumente zur Schadensvorbeugung.

Mit dem Modellprojekt der DGT soll auch geklärt werden, ob bestimmte Anforderungen an die medizinische Erfahrung die Risiken mindern. Die Abhängigkeit der Operationsletalität vom Hospitalvolumen ist für die Thoraxchirurgie wissenschaftlich gesichert [1]. Es steht bereits fest, dass Qualitätsaspekte künftig stärker als bisher bei Steuerungsentscheidun- gen und bei der Vergütung in deutschen Krankenhäusern berücksichtigt werden sollen [2]. Die Gestaltungshoheit insbesondere auch für die Qualitätssicherung im Krankenhaus besitzt der Gemeinsame Bundesausschuss (G-BA). Mindestmengen für bestimmte planbare Leistungen spielen in diesem Zusammenhang eine wesentliche Rolle. So gilt im Bereich der Chirurgie/Orthopädie seit dem 01.01.2015 wieder die jährliche Mindestmenge von 50 für den Einsatz eines künstlichen Kniegelenks (Kniegelenk-Totalendoprothese/ Knie-TEP). Für die Thoraxchirurgie sind Mindestmengen ebenso notwendig, um über den entsprechenden Übungs- und Erfahrungseffekt ein ausreichendes Komplikationsmanagement vorhalten zu können und somit niedere Operationsletalitäten zu erzielen.

Trotz aller gesetzlichen und prophylaktischen Maßnahmen lassen sich Komplikationen bei chirurgischen Eingriffen nicht vollständig ausschließen. Die Erkennung und Beherrschung der Komplikation erfordert sehr viel klinische Erfahrung. Thoraxchirurgisches Komplikationsmanagement wird in Lehrbüchern kaum vermittelt. Umso wichtiger sind die nachfolgenden Arbeiten (Lehrbeiträge), die alle von sehr erfahrenen Thoraxchirurgen angefertigt wurden. Der Themenkomplex des thoraxchirurgischen Komplikationsmanagements behandelt neue Aspekte in der Behandlung alt bekannter Probleme, so z. B. die endoskopische Versorgung von Trachealeinrissen durch eine atraumatische endotracheale Naht ( $S$. Welter, Essen). Es werden aber auch Komplikationen und ihre Vermeidung bzw. Beherrschung dargestellt, die sich erst in den letzten Jahren durch z. B. neue medikamentöse Therapie ergeben haben. So berichtet $R$. Scheubel (Wangen) in seinem Beitrag zu blutgerinnungsbeinflussenden Medikamenten in der Thoraxchirurgie über das perioperative Management von Patienten mit den neuen oralen Antikoagulationen (z. B. Dabigratan, Rivaroxaban, Apixaban).

Herr Kollege L. Lampl (Augsburg) weist aber auch explizit daraufhin, dass den eigenen Erfahrung Grenzen gesetzt sein können. Hier ist dann die Expertise anderer chirurgischer Fächer gefragt, die im Vorfeld schwieriger Eingriffe auch eingeplant werden sollte. Die Maxime für jeden ordentlichen Chirurgen ist und bleibt: „Salus aegroti suprema lex“ („Das Heil des Kranken sei höchstes Gesetz; [3]).

\section{Korrespondenzadresse}

Prof. Dr. H.S. Hofmann

Abteilung für Thoraxchirurgie,

Universitätsklinikum

Regensburg

Franz-Josef-Strauss-Allee 11, 93053 Regensburg hans-stefan.hofmann@t-

online.de

Interessenkonflikt. H.S. Hofmann gibt an, dass kein Interessenkonflikt besteht.

\section{Literatur}

1. Kaiser D (2007) Minimum quantities from a thoracic surgical standpoint. Chirurg 78(11):1012-1017

2. Gröhe H (2014) Begrüßungsworte auf der 6. Qualitätssicherungskonferenz des Gemeinsamen Bundesausschusses (G-BA), Berlin 22.09.2014

3. Lampl L (2015) Vorbeugung und Behandlung intraoperativer Komplikationen bei thoraxchirurgischen Eingriffen. Chirurg 